\title{
Impact of osteoporosis and vertebral fractures on quality-of-life. a population-based study in Valencia, Spain (The FRAVO Study)
}

\author{
José Sanfélix-Genovés ${ }^{1,2^{*}}$, Isabel Hurtado ${ }^{1}$, Gabriel Sanfélix-Gimeno ${ }^{1}$, Begoña Reig-Molla ${ }^{3}$ and Salvador Peiró ${ }^{1}$
}

\begin{abstract}
Background: To describe the health related quality of life in a population sample of postmenopausal women over the age of 50 and resident in the city of Valencia (Spain), according to the presence/absence of osteoporosis and the severity of prevalent morphometric vertebral fractures.

Methods: A cross-sectional age-stratified population-based sample of 804 postmenopausal women of 50 years of age and older were assessed with the SF-12 questionnaire. Information about demographic features, lifestyle, clinical features, educational level, anti-osteoporotic and other treatments, comorbidities and risk factors for osteoporosis were collected using an interviewer-administered questionnaire and densitometric evaluation of spine and hip and spine $x$-rays were carried out.
\end{abstract}

Results: In the non-adjusted analysis, mild and moderate-severe vertebral fractures were associated with decreased scores in the SF-12 Physical Component Summary (PCS) but not in the Mental Component Summary (MCS), while densitometric osteoporosis with no accompanying fracture was not associated with a worse health related quality of life. In multivariate analysis worse PCS scores were associated to the age groups over 70 (-2.43 for 70-74 group and -2.97 for 75 and older), chronic conditions $(-4.66,-6.79$ and -11.8 according to the presence of 1,2 or at least 3 conditions), obesity (-5.35), peripheral fracture antecedents (-3.28), hypoestrogenism antecedents $(-2.61)$ and the presence of vertebral fracture (-2.05).

Conclusions: After adjusting for confounding factors, the physical components of health related quality of life were significantly lower in women with prevalent osteoporotic vertebral fractures than in women -osteoporotic or not- without vertebral fractures.

\section{Introduction}

Osteoporosis is a common condition characterized by decreased bone mass and increased susceptibility to fractures [1]. The most common clinical complications of osteoporosis are hip, wrist, and vertebral fractures. Vertebral fractures (VFX) are the most prevalent osteoporosis-related fractures but they are often asymptomatic, and their underdiagnosis and undertreatment is well documented $[2,3]$.

Measures of Health Related Quality of Life (HRQoL) have gained increasing attention as relevant outcomes in clinical studies of osteoporosis $[4,5]$. These measures are

\footnotetext{
* Correspondence: sanfelix_jos@gva.es

${ }^{1}$ Centro Superior de Investigación en Salud Pública (CSISP), Valencia, Spain Full list of author information is available at the end of the article
}

also used in epidemiological surveys, complementary to data on morbidity and health care utilization, to estimate the burden of disease and often to compare with other chronic diseases. Several instruments, both generic and disease targeted, have been used to examine HRQoL in osteoporosis and osteoporotic fractures [5-7]. The specific instruments most widely used include the Osteoporosis Quality of Life Questionnaire (OQLQ) [6,7] and its reduced version the mini-OQLQ [8], the Quality of Life Questionnaire of the European Foundation for Osteoporosis (QUALEFFO) $[9,10]$, the Osteoporosis Assessment Questionnaire (OPAQ) [11,12], the Osteoporosis-Targeted Quality of Life Questionnaire (OPTQoL) $[13,14]$ and the assessment of health-related quality of life in osteoporosis (ECOS-16) [15]. Among the generic instruments, those most used in osteoporotic

\section{Ciomed Central}


patients includes the EuroQol 5-D (EQ5D) $[16,17]$, the Medical Outcomes Study Survey Form (MOS-SF) in its SF-12 [18] or SF-36 [16] versions that could be combined with the disease-specific module Quality of Life in Osteoporosis (QUALIOST) $[19,20]$, and the Health Utility Index $[7,21]$.

Vertebral fractures and deformities result in back pain, disability, limitations in physical functioning and psychosocial impairment [22]. An increasing amount of literature has shown the relation between prevalent VFX (their number, severity and, occasionally, lumbar localization) and HRQoL decline [5,18,23-26]. Lower HRQoL has also been associated with incident VFX, with or without clinical manifestations [5,27-29]. However, the association with osteoporosis in the absence of fracture or with only mild morphometric fractures has been less studied. The aim of this study is to describe the HRQoL in a population sample of postmenopausal women of 50 years old and over and resident in the city of Valencia (Spain), according the presence/absence of osteoporosis and the severity of prevalent morphometric vertebral fractures.

\section{Methods \\ Design}

Population-based cross-sectional study conducted between February 2006 and March 2007, designed primarily to estimate the prevalence of densitometric osteoporosis and vertebral fracture.

\section{Population and simple}

The study's population was post-menopausal women over the age of 50 living in the city of Valencia, Spain, excluding women with cognitive impairment, physical impediments preventing women from going to the radiology centre by her own means, race other than Caucasian and unwillingness to participate in the study. The methods of the FRAVO study, mainly designed to estimate the population prevalence of vertebral fracture and densitometric osteoporosis, have been fully described elsewhere [30]. Briefly, 1,758 women were selected from a simple age-stratified (50-54, 55-59, 60-64, 65-69, 70-74 y 75+) random sample from among the residents of Valencia, and invited to participate in the study. Only 1,314 confirmed receipt of the letter $(74.7 \%)$ and of these, 76 presented at least one exclusion criteria, 371 declined to participate and 43 did not keep their appointments for the examinations, leaving 824 women participating in the study. In 19 cases the spine $x$-ray or the densitometry was not available and in 1 case the HRQoL questionnaire was not entirely fulfilled, leaving 804 women for analysis (dropouts by reason and age groups are described in Additional file 1).

\section{Main outcome measure}

Health related quality of life was measured with the Spanish version-2 of the MOS SF-12 questionnaire [31], a simplified self-administered version of the SF-36 that could be completed within two minutes. The SF-12 is a generic instrument consisting of 12 items covering the domains of physical functioning, role limitations due to physical health problems, bodily pain, general health, vitality, social functioning, role limitations due to emotional problems and mental health. These domains can be summarized into a physical component summary scale (PCS-12) and a mental component summary scale (MCS-12). In the SF-12 version-2 for each one of the 8 domains and the summary components, items are aggregated and transformed into a 0 to 100 score, a low score indicating a lower HRQoL. To facilitate interpretation, the PCS and MCS scores are standardized with population norms, 50 (SD: 10) being the average of the general population [31]. Because Spanish weights were not available for the SF-12v2 at the time of analysis, we use the North American weights. Figures higher or lower than 50 should be interpreted as better or worse HRQoL scores than the reference population.

\section{Other variables and definitions}

Information about demographic features, lifestyle, clinical features, educational level, anti-osteoporotic and other treatments, comorbidities and risk factors for osteoporosis was collected using an intervieweradministered questionnaire. Among other variables, it included the subject's age, place of birth, educational level (no studies, primary, secondary/university, and unknown studies), obesity grade II or more (body mass index $(\mathrm{BMI})>35$ ), hypoestrogenism antecedents (menopause before age 40 and/or amenorrhea for more than a year) and asked whether the subject had a history of osteoporotic fracture excluding major traumatisms in any location. Using the information on risk factors, comorbidities and treatments, we constructed a variable to account for the presence of chronic conditions that could affect the HRQoL: taking corticoids for at least 3 months in the last year, gait abnormalities for any reason (or postural instability, impaired balance or anticonvulsive treatment), cognitive or visual deficit, depression (or taking lithium), and specific self-referred conditions such as gastrectomy, bowel resection, inflammatory bowel disease, thyroidectomy (or taking thyroxin), diabetes mellitus, chronic liver diseases, chronic obstructive pulmonary disease, rheumatoid arthritis, chronic kidney failure and transplantation (or immunosuppressive treatment).

Spine radiographs were performed using standardized techniques and two radiologists, blinded to all data 
concerning the patients, performed the semiquantitative evaluation of the radiographs using the Genant method [32] to standardize the diagnosis of fractures. Each vertebrae, including $\mathrm{T} 4$ to $\mathrm{L} 4$, were classified into one of the five grades on Genant's score. Densitometric examinations were performed with two calibrated densitometers (Dual-energy X-ray absorptiometry or DXA central) for the lumbar spine and the femoral neck. The World Health Organization definitions [33] of osteopenia and of osteoporosis were applied in both locations and the greater value was taken into account.

\section{Ethical Aspects}

The study was approved by the Ethics Committee for Clinical Research of the Primary Care Departments of Valencia and Castellon (Regional Government of Valencia Department of Health). All of the participating women were informed of the study's characteristics and risks (basically, those associated with exposure to $\mathrm{x}$-rays), and all gave signed informed consent prior to examination. Because the study data could be clinically useful, we communicated the results of the densitometric and $x$-ray examinations to the patients, with a recommendation to visit their primary care doctor when pertinent.

\section{Analysis}

First, we describe the socio-demographic and clinical characteristics of the sample according to the following 4 groups: 1) absence of VFX without densitometric osteoporosis, 2) absence of VFX with densitometric osteoporosis, 3) presence of only mild VFX Genant grade 1, and 4) presence of moderate-severe VFX Genant grade 2-3. Chi-square (or Fisher exact test when pertinent) was used to assess differences among groups. Second, we perform a descriptive analysis of the PCS and MCS scores stratified by groups and characteristics of the sample. To assess the possible differences between groups Multivariable Analysis of Variance (MANOVA) was used. The relevant $\mathrm{p}$-value in this analysis (variance between groups) was specified as $\mathrm{p}$ (groups) in the corresponding tables. Because it provides helpful information, p-values corresponding to the variance between levels of the corresponding independent variable, specified as $\mathrm{p}$ (variable name), were also included in the tables. Third, we estimate means and confidence intervals $(95 \% \mathrm{CI})$ of the SF12 domains and the PCS and MCS scores for the 4 groups, and use the ANOVA Oneway methods to evaluate differences between groups. Totals for SF-12 domains and summary scores were weighted to represent the population agestructure of the Valencia city. Finally, we use multivariate regression analysis to analyze the independent effects of VFX and osteoporosis on the PCS scores, controlling the effect of different covariables (age, chronic conditions, obesity, hypoestrogenism antecedents, fracture antecedents and educational level). We constructed an initial model with all relevant variables and we used the backward-stepwise technique, with a removing probability of 0.10 and an entry probability of 0.05 , to retain the significant factors. All analyses were performed using the STATA 10.0 (Stata Corp., College Station, Texas) statistical software.

\section{Results}

Clinical and demographic characteristics of the participating women according to the four predefined groups of absence (with or without densitometric osteoporosis) or presence of VFX (mild or moderate-severe) in the $\mathrm{x}$-ray are shown in Table 1 . Relevant characteristics of the sample included $51.9 \%$ of women with densitometric osteopenia and $28.0 \%$ with densitometric osteoporosis, $72.9 \%$ with at least one chronic condition, $22.1 \%$ with antiosteoporotic treatment, and 15.6\% (mild: 9.4\%; moderate-severe: $6.2 \%$ ) with radiological vertebral fractures (21.4\% weighting the sample by the age structure of the city of Valencia). Vertebral fracture was most prevalent with older age groups, lower educational level, densitometric osteoporosis, self-referred antecedents of non-vertebral clinical fracture, and in women with antiosteoporotic treatment.

PCS scores by the women's characteristics and groups are shown in Table 2. PCS scores decreased with age (from 48.5 in the 50-54 years group to 40.4 in the 75 and older group), number of chronic conditions (from 50.6 for no comorbidities to 36.9 in people with 3 or more chronic conditions), antecedents of non-vertebral fracture, hypoestrogenism antecedents, obesity, antiosteoporotic treatment, and lumbar or both thoracic and lumbar localization, and increased with educational level. PCS scores also decreased with the presence of vertebral fracture (mild: 41.6, and moderate-severe: 40.3, vs. 45.6 and 46.2 in the groups without VFX). MCS scores (Table 3 ) were only affected by chronic conditions (worse with more conditions) and obesity (better in women with BMI higher than 35).

Women's scores in the eight SF-12 domains and both summary components (total are weighted by the age structure of the Valencia female population) are shown in Table 4. Physical functioning (more than 65 in woman without fracture vs. 44 in women with moderate-severe fracture), physical role, social functioning, general health, emotional role and PCS showed statistically significant differences, usually between the moderate-severe VFX group and groups without fracture. The densitometric osteoporotic group did not show differences between groups with normal-osteopenia densitometry. The domains of bodily pain, vitality mental 
Table 1 Clinical and socio-demographic characteristics of the sample by osteoporosis and morphometric vertebral fracture (\%)

\begin{tabular}{|c|c|c|c|c|c|}
\hline & \multicolumn{2}{|c|}{ Without vertebral fracture } & \multicolumn{2}{|c|}{ With Vertebral fracture } & \multirow[t]{2}{*}{ Total } \\
\hline & $\begin{array}{c}\text { T-Score } \\
>-2.5\end{array}$ & $\begin{array}{c}\text { T-Score } \\
\leq-2.5\end{array}$ & Mild & Moderate-severe & \\
\hline \multicolumn{6}{|c|}{ Age group $(p<0.001)$} \\
\hline - $50-54$ years & $86(79.6)$ & $17(15.7)$ & $3(2.8)$ & $2(1.8)$ & $108(13.4)$ \\
\hline - 55-59 years & $118(77.6)$ & $23(15.1)$ & $7(4.6)$ & $4(2.6)$ & $152(18.9)$ \\
\hline - 60-64 years & $117(69.2)$ & $32(18.9)$ & $17(10.1)$ & $3(1.8)$ & $169(21.0)$ \\
\hline - $65-69$ years & 99 (59.6) & $43(25.9)$ & $14(8.4)$ & $10(6.0)$ & $166(20.6)$ \\
\hline - $70-74$ years & $68(47.6)$ & $40(28.0)$ & $20(14.0)$ & $15(10.5)$ & $143(17.8)$ \\
\hline - 75 years and older & $22(33.3)$ & $13(19.7)$ & $15(22.7)$ & $16(24.2)$ & $66(8.2)$ \\
\hline \multicolumn{6}{|c|}{ Educational level $(p<0.001)$} \\
\hline - Without studies & $79(52.3)$ & $26(17.2)$ & $25(16.6)$ & $21(13.9)$ & $151(18.8)$ \\
\hline - Primary & $215(62.5)$ & $82(23.8)$ & $28(8.1)$ & $19(5.5)$ & $344(42.8)$ \\
\hline - Second./university & $132(69.1)$ & $44(23.0)$ & $11(5.8)$ & $4(2.1)$ & $191(23.8)$ \\
\hline - Unknown & $84(71.2)$ & $16(13.6)$ & $12(10.2)$ & $6(5.1)$ & $118(14.6)$ \\
\hline \multicolumn{6}{|c|}{ Densitometry $(p<0.001)$} \\
\hline - Normal & $146(90.1)$ & $0(0.0)$ & $12(7.4)$ & $4(2.5)$ & $162(20.1)$ \\
\hline - Osteopenia & $364(87.3)$ & $0(0.0)$ & $32(7.7)$ & $21(5.0)$ & $417(51.9)$ \\
\hline - Osteoporosis & $0(0.0)$ & $168(74.7)$ & $32(14.2)$ & $25(11.1)$ & $225(28.0)$ \\
\hline \multicolumn{6}{|c|}{ Chronic conditions $(p=0.094)^{*}$} \\
\hline - None & $150(68.8)$ & $49(22.5)$ & $11(5.0)$ & $8(3.7)$ & $218(27.1)$ \\
\hline-1 & $176(61.8)$ & $62(21.7)$ & $30(10.5)$ & $17(6.0)$ & $285(35.5)$ \\
\hline-2 & $118(63.4)$ & $33(17.7)$ & $19(10.2)$ & $16(8.6)$ & $186(23.1)$ \\
\hline - 3 or more & $66(57.4)$ & $24(20.9)$ & $16(13.9)$ & $9(7.8)$ & $115(14.3)$ \\
\hline \multicolumn{6}{|c|}{ Antecedents of non-vertebral fracture $(p=0.020)$} \\
\hline - No & $493(64.6)$ & $156(20.4)$ & $69(9.0)$ & $45(5.9)$ & $763(94.9)$ \\
\hline - Yes & $17(41.5)$ & $12(29.3)$ & $7(17.1)$ & $5(12.2)$ & $41(5.1)$ \\
\hline \multicolumn{6}{|c|}{ Hypoestrogenism antecedents $(p=0.407)$} \\
\hline - No & $416(64.0)$ & $131(20.1)$ & $65(10.0)$ & $38(5.8)$ & $650(80.8)$ \\
\hline - Yes & $94(61.0)$ & $37(24.0)$ & $11(7.1)$ & $12(7.8)$ & $154(19.1)$ \\
\hline \multicolumn{6}{|c|}{ Obesity BMI>35 ( $p=0.010)$} \\
\hline - No & $447(62.0)$ & $162(22.5)$ & $69(9.6)$ & $43(6.0)$ & $721(89.7)$ \\
\hline - Yes & $63(75.9)$ & $6(7.2)$ & $7(8.4)$ & $7(8.4)$ & $83(10.3)$ \\
\hline \multicolumn{6}{|c|}{ Antiosteoporotic treatment $(p<0.001)$} \\
\hline - No & $416(66.4)$ & $119(19.0)$ & $62(9.9)$ & $29(4.6)$ & $626(77.9)$ \\
\hline - Yes & $94(52.8)$ & $49(27.5)$ & $14(7.9)$ & $21(11.8)$ & $178(22.1)$ \\
\hline \multicolumn{6}{|c|}{ Vertebral fracture localization $(p<0.001)$} \\
\hline - Thoracic & - & - & $65(71.4)$ & $26(28.6)$ & $91(72.2)$ \\
\hline - Lumbar & - & - & $8(61.5)$ & $5(38.5)$ & $13(10.3)$ \\
\hline - Both & - & - & $3(13.6)$ & 19(86.4) & $22(17.5)$ \\
\hline TOTAL & $510(63.4)$ & $168(20.9)$ & $76(9.4)$ & $50(6.2)$ & $804(100)$ \\
\hline
\end{tabular}

All percentages by rows except in the total column (by columns). BMI: Body Mass Index. *Chronic conditions: corticoid treatment, gait abnormalities for any reason, cognitive or visual deficit, depression, gastrectomy, bowel resection, inflammatory bowel disease, thyroidectomy, diabetes mellitus, chronic liver diseases, chronic obstructive pulmonary disease, rheumatoid arthritis, chronic kidney failure and transplantation. p-values correspond to Pearson's chi-squared test. 
Table 2 Physical component summary score by population characteristics

\begin{tabular}{|c|c|c|c|c|c|}
\hline & \multicolumn{2}{|c|}{ Without vertebral fracture } & \multicolumn{2}{|c|}{ With Vertebral fracture } & \multirow[t]{2}{*}{ Total } \\
\hline & T-Score $>-2.5$ & T-Score $\leq-2.5$ & Mild & Mod-severe & \\
\hline \multicolumn{6}{|c|}{ Age group $[p($ model $)<0.0001 ; p($ age $)<0.0001 ; p$ (groups) $=0.0405]$} \\
\hline - 50-54 years & 48.02 & 51.50 & 51.93 & 36.86 & 48.46 \\
\hline$-55-59$ years & 48.65 & 46.11 & 43.14 & 50.21 & 48.05 \\
\hline - $60-64$ years & 44.85 & 48.72 & 42.49 & 41.73 & 45.29 \\
\hline$-65-69$ years & 43.19 & 44.57 & 41.51 & 42.76 & 43.38 \\
\hline - $70-74$ years & 41.92 & 42.76 & 39.59 & 37.26 & 41.34 \\
\hline - 75 years and older & 39.55 & 42.97 & 40.67 & 39.15 & 40.38 \\
\hline \multicolumn{6}{|c|}{ Educational level $[p($ model $)<0.0001 ; p($ educational level) $<0.0001 ; p($ groups $)=0.0265]$} \\
\hline - Without studies & 40.44 & 42.23 & 39.92 & 36.52 & 40.12 \\
\hline - Primary & 44.84 & 46.29 & 42.77 & 42.04 & 44.84 \\
\hline - Second./university & 48.04 & 47.69 & 45.36 & 37.19 & 47.58 \\
\hline - Unknown & 46.87 & 43.01 & 39.74 & 49.68 & 45.76 \\
\hline \multicolumn{6}{|c|}{ Densitometry $[p($ model $)<0.0028 ; p$ (densitometry) $<0.9419 ; p$ (groups) $=0.0004]$} \\
\hline - Normal & 45.37 & - & 38.44 & 47.77 & 44.91 \\
\hline - Osteopenia & 45.31 & - & 41.32 & 40.11 & 44.74 \\
\hline - Osteoporosis & - & 45.72 & 43.11 & 39.17 & 44.62 \\
\hline \multicolumn{6}{|c|}{ 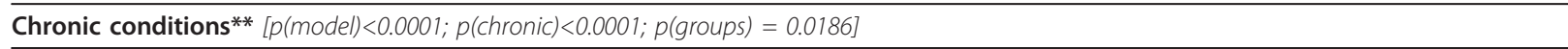 } \\
\hline - None & 50.61 & 50.78 & 54.00 & 45.49 & 50.63 \\
\hline-1 & 46.34 & 45.43 & 38.41 & 43.01 & 45.11 \\
\hline-2 & 42.30 & 44.90 & 40.96 & 36.65 & 42.14 \\
\hline - 3 or more & 36.04 & 37.27 & 39.93 & 36.82 & 36.90 \\
\hline \multicolumn{6}{|c|}{ Antecedents of non-vertebral fracture $[p($ model $)<0.0058 ; p$ (non-vert. fract $)<0.0001 ; p$ (groups) $=0.0010]$} \\
\hline- No & 45.56 & 46.14 & 41.52 & 40.37 & 45.01 \\
\hline - Yes & 38.40 & 40.16 & 42.64 & 39.14 & 39.73 \\
\hline \multicolumn{6}{|c|}{ Hypoestrogenism antecedents $[p($ model $)<0.0001 ;$ p(hypoestrogenism $)<0.0001 ; p($ groups $)=0.0003]$} \\
\hline- No & 46.10 & 46.37 & 41.50 & 41.59 & 45.43 \\
\hline - Yes & 41.88 & 43.42 & 42.37 & 36.03 & 41.83 \\
\hline \multicolumn{6}{|c|}{ Obesity BMI>35 [p(model)<0.0001; $p$ (obesity) $<0.0001 ; p$ (groups) $=0.0005]$} \\
\hline- No & 46.34 & 46.17 & 41.68 & 40.77 & 45.52 \\
\hline - Yes & 38.14 & 33.65 & 41.12 & 37.09 & 37.98 \\
\hline \multicolumn{6}{|c|}{ Antiosteoporotic treatment $[p($ model $)<0.0001 ; p($ treatment $)<0.0143 ; p$ (groups $)=0.0008]$} \\
\hline - No & 45.65 & 46.19 & 42.45 & 42.34 & 45.28 \\
\hline - Yes & 43.87 & 44.57 & 37.96 & 37.37 & 42.83 \\
\hline \multicolumn{6}{|c|}{ Vertebral fracture localization $[p($ model $)=0.0700 ; p($ localization $)=0.0375 ; p($ groups $)=0.7755]$} \\
\hline - Thoracic & - & - & 42.83 & 42.24 & 42.66 \\
\hline - Lumbar & - & - & 34.61 & 40.19 & 36.76 \\
\hline - Both & - & - & 34.12 & 37.56 & 37.09 \\
\hline \multicolumn{6}{|c|}{ Total [p(groups) $=0.0004]$} \\
\hline TOTAL & 45.33 & 45.72 & 41.62 & 40.26 & $44.14^{*}$ \\
\hline
\end{tabular}

*Total weighted to represent the distribution of the female population by age in the city of Valencia.

**Chronic conditions: corticoid treatment, gait abnormalities for any reason, cognitive or visual deficit, depression, gastrectomy, bowel resection, inflammatory bowel disease, thyroidectomy, diabetes mellitus, chronic liver diseases, chronic obstructive pulmonary disease, rheumatoid arthritis, chronic kidney failure and transplantation. BMI: Body Mass Index.

$p$-values correspond to the multivariate analysis of variance (MANOVA). 
Table 3 Mental component summary score by population characteristics

\begin{tabular}{|c|c|c|c|c|c|}
\hline & \multicolumn{2}{|c|}{ Without vertebral fracture } & \multicolumn{2}{|c|}{ With Vertebral fracture } & \multirow[t]{2}{*}{ Total } \\
\hline & T-Score $>-2.5$ & T-Score $\leq-2.5$ & Mild & Mod-severe & \\
\hline \multicolumn{6}{|c|}{ Age group $[p($ model $)=0.3440 ; p($ age $)=0.6394 ; p($ groups $)=0.1509]$} \\
\hline - 50-54 years & 46.84 & 43.99 & 42.70 & 39.31 & 46.14 \\
\hline$-55-59$ years & 45.76 & 43.89 & 43.85 & 53.67 & 45.60 \\
\hline - $60-64$ years & 45.42 & 45.52 & 47.90 & 46.19 & 45.70 \\
\hline - $65-69$ years & 44.85 & 43.43 & 49.42 & 37.21 & 44.41 \\
\hline - $70-74$ years & 45.86 & 44.38 & 44.55 & 48.36 & 45.52 \\
\hline - 75 years and older & 44.61 & 41.24 & 45.70 & 46.72 & 44.77 \\
\hline \multicolumn{6}{|c|}{ Educational level $[p($ model $)=0.1164 ; p($ educ $)=0.2030 ; p$ (groups $)=0.1340]$} \\
\hline - Without studies & 45.08 & 42.04 & 45.53 & 45.98 & 44.76 \\
\hline - Primary & 45.52 & 43.28 & 45.25 & 44.09 & 44.90 \\
\hline - Second./university & 46.01 & 46.07 & 46.62 & 47.45 & 46.09 \\
\hline - Unknown & 45.97 & 45.20 & 49.97 & 47.26 & 46.34 \\
\hline \multicolumn{6}{|c|}{ Densitometry $[p($ model $)=0.0561 ; p($ densito $)=0.0753 ; p($ groups $)=0.4137]$} \\
\hline - Normal & 45.15 & - & 46.97 & 51.09 & 45.43 \\
\hline - Osteopenia & 45.86 & - & 47.73 & 46.84 & 46.06 \\
\hline - Osteoporosis & - & 44.01 & 44.59 & 43.56 & 44.04 \\
\hline \multicolumn{6}{|c|}{ Chronic conditions $^{* *}[p($ model $)<0.0001 ; p($ chronic $)<0.0001 ; p($ groups $)=0.0354]$} \\
\hline - None & 48.14 & 46.00 & 48.48 & 49.81 & 47.74 \\
\hline-1 & 46.22 & 45.39 & 48.50 & 45.97 & 46.27 \\
\hline-2 & 45.06 & 42.18 & 46.27 & 47.79 & 44.90 \\
\hline - 3 or more & 39.59 & 38.90 & 40.67 & 36.95 & 39.39 \\
\hline \multicolumn{6}{|c|}{ Antecedents of non-vertebral fracture $[p($ model $)=0.2081 ; p($ antec $)=0.5708 ; p$ (groups) $=0.1379]$} \\
\hline$-\mathrm{No}$ & 45.59 & 44.45 & 45.75 & 46.10 & 45.41 \\
\hline - Yes & 47.38 & 38.20 & 51.53 & 40.47 & 44.57 \\
\hline \multicolumn{6}{|c|}{ Hypoestrogenism antecedents $[p($ model $)=0.1251 ; p$ (hypoes $)=0.2038 ; p($ groups $)=0.1181]$} \\
\hline- No & 45.35 & 44.02 & 46.67 & 44.95 & 45.19 \\
\hline - Yes & 47.01 & 43.95 & 44.04 & 47.91 & 46.09 \\
\hline \multicolumn{6}{|c|}{ Obesity BMI>35 [p(model) = 0.0303; $p$ (obes) $=0.0242 ; p$ (groups) $=0.2067]$} \\
\hline$-\mathrm{No}$ & 45.33 & 43.98 & 46.33 & 45.08 & 45.11 \\
\hline - Yes & 47.95 & 44.82 & 45.94 & 48.39 & 47.59 \\
\hline \multicolumn{6}{|c|}{ Antiosteoporotic treatment $[p($ model $)=0.2264 ; p($ treatment $)=0.8042 ; p$ (groups $)=0.1425]$} \\
\hline- No & 45.63 & 44.07 & 46.65 & 45.68 & 45.44 \\
\hline - Yes & 45.76 & 43.83 & 44.67 & 45.34 & 45.10 \\
\hline \multicolumn{6}{|c|}{ Vertebral fracture localization $[p($ model $)=0.7076 ; p($ loc $)=0.5582 ; p$ (groups $)=0.3955]$} \\
\hline - Thoracic & - & - & 46.60 & 44.01 & 45.86 \\
\hline - Lumbar & - & - & 44.49 & 45.47 & 44.87 \\
\hline - Both & & & 44.41 & 47.66 & 47.22 \\
\hline \multicolumn{6}{|c|}{ Total $[p$ (groups) $=0.1330]$} \\
\hline TOTAL & 45.66 & 44.01 & 46.29 & 45.54 & $45.29^{*}$ \\
\hline
\end{tabular}

*Total weighted to represent the distribution of the female population by age in the city of Valencia.

**Chronic conditions: corticoid treatment, gait abnormalities for any reason, cognitive or visual deficit, depression, gastrectomy, bowel resection, inflammatory bowel disease, thyroidectomy, diabetes mellitus, chronic liver diseases, chronic obstructive pulmonary disease, rheumatoid arthritis, chronic kidney failure and transplantation. BMI: Body Mass Index.

$p$ values correspond to the multivariate analysis of variance (MANOVA). 
Table 4 SF-12 domains and summary scores by presence or absence of osteoporosis and morphometric vertebral fracture

\begin{tabular}{|c|c|c|c|c|c|c|}
\hline & & \multicolumn{2}{|c|}{ Without vertebral fracture } & \multicolumn{2}{|c|}{ With Vertebral fracture } & \multirow[t]{2}{*}{ Total* } \\
\hline & & T-Score $>-2.5$ & T-Score $\leq-2.5$ & Mild & Mod-severe & \\
\hline \multicolumn{2}{|c|}{ Physical functioning } & 65.05 & 65.62 & 57.24 & 44 & 60.46 \\
\hline & $p<0.0001$ & $(62.14-67.96)$ & $(60.21-71.04)$ & $(49.22-65.25)$ & $(34.53-53.47)$ & $(57.45-63.48)$ \\
\hline \multirow[t]{2}{*}{ Physical role } & & 80.78 & 78.57 & 71.71 & 69.5 & 77.1 \\
\hline & $p=0.0003$ & (78.83-82.74) & $(74.99-82.14)$ & $(65.88-77.54)$ & $(62.91-76.08)$ & $(75.09-79.10)$ \\
\hline \multirow[t]{2}{*}{ Bodily pain } & & 74.61 & 74.85 & 70.06 & 70 & 74.17 \\
\hline & $p=0.3741$ & (70.65-79.04) & $(70.65-79.04)$ & $(63.53-81.4)$ & $(61.63-78.36)$ & $(71.87-76.47)$ \\
\hline \multirow[t]{2}{*}{ General health } & & 49.24 & 49.13 & 40.13 & 46 & 47.57 \\
\hline & $p=0.0146$ & $(47.24-51.24)$ & $(45.50-52.77)$ & $(34.15-46.11)$ & $(39.00-52.99)$ & $(45.62-49.51)$ \\
\hline \multirow[t]{2}{*}{ Vitality } & & 22.15 & 22.17 & 27.3 & 19 & 21.94 \\
\hline & $p=0.3147$ & $(19.86-24.45)$ & $(18.36-25.97)$ & $(20.82-33.77)$ & $(11.88-26.11)$ & $(19.79-24.10)$ \\
\hline \multicolumn{2}{|c|}{ Social functioning } & 86.27 & 82.29 & 77.96 & 74.5 & 81.98 \\
\hline & $p=0.0003$ & $(81.2-87.5)$ & (78.50-86.07) & $(71.49-84.42)$ & $(66.97-82.02)$ & $(79.80-84.17)$ \\
\hline \multirow[t]{2}{*}{ Emotional role } & & 86.37 & 82.66 & 83.22 & 80 & 84.19 \\
\hline & $p=0.0250$ & (84.78-87.95) & (79.76-85.56) & (78.36-88.08) & $(73.95-86.04)$ & $(82.47-85.91)$ \\
\hline \multirow[t]{2}{*}{ Mental Health } & & 56.91 & 54.61 & 58.55 & 58.25 & 56.87 \\
\hline & $p=0.4418$ & $(55.11-58.70)$ & $(51.46-57.75)$ & $(53.90-63.19)$ & $(52.85-63.64)$ & $(55.08-58.66)$ \\
\hline \multirow[t]{2}{*}{$\overline{P C S}$} & & 45.32 & 45.72 & 41.62 & 40.25 & 44.14 \\
\hline & $p=0.0004$ & $(44.41-46.23)$ & $(44.01-47.43)$ & $(39.07-44.17)$ & $(37.25-43.25)$ & (43.24-45.05) \\
\hline \multirow[t]{2}{*}{ MCS } & & 45.65 & 44 & 46.29 & 45.54 & 45.29 \\
\hline & $p=0.1330$ & $(44.90-46.41)$ & $(42.69-45.32)$ & $(44.32-48.25)$ & $(43.00-48.08)$ & $(44.54-46.06)$ \\
\hline
\end{tabular}

SF-12: Medical Outcomes Study Survey Form 12; PCS: Physical Component Summary; MCS: Mental Component Summary. *Total weighted to represent the distribution of the female population by age in the city of Valencia.

health and the MCP score did not show differences among groups.

Results of the multivariable regression fitted to explore the independent relationship between the PCS score and VFX controlling the effect of possible confounders are shown in Table 5. From a constant of 51.83, PCS scores decrease with age groups older than 70 (-2.43 for 70-74 group and -2.97 for 75 and older), chronic conditions $(-4.66,-6.79$ and -11.8 according to the presence of 1,2 or at least 3 conditions), BMI $>35(-5.35)$, peripheral fracture antecedents $(-3.28)$, and hypoestrogenism antecedents $(-2.61)$. Controlling the effect of these variables, the presence of VFX (any grade) was independently associated with a reduction of -2.05 in the PCS score.

\section{Discussion}

In the bivariate analysis (not adjusted) mild and moderate-severe vertebral fractures were associated with a decreased HRQoL measured by the SF-12 Physical Component Summary score but not with the Mental Component Summary score, while densitometric osteoporosis with no accompanying fracture was not associated with any deterioration in HRQoL. Multivariate analysis, controlling by several confounders including age and comorbidities, retained the association between vertebral fracture and worse physical HRQoL. These results confirm that prevalent morphometric vertebral fractures are independently associated with lower scores in the physical domains of HRQoL. On the contrary, and as expected, densitometric osteoporosis without accompanying fracture was not related with HRQoL physical scores.

Table 5 Factors associated with Physical Component Summary score in women of $\mathbf{5 0}$ years and older.

\begin{tabular}{llccc}
\hline & & Coef. & $\mathbf{9 5 \%} \mathbf{C l}$ & $\mathbf{p}$ \\
\hline Age group & 70-74 years & -2.43 & $-4.24 ;-0.62$ & 0.009 \\
\cline { 3 - 6 } & 75 years and older & -2.97 & $-5.53 ;-0.41$ & 0.023 \\
\hline Chronic conditions* & 1 & -4.66 & $-6.36 ;-2.95$ & $<0.001$ \\
\cline { 3 - 5 } & 2 & -6.79 & $-8.73 ;-4.86$ & $<0.001$ \\
\cline { 3 - 6 } & 3 or more & -11.48 & $-13.74 ;-9.23$ & $<0.001$ \\
\hline Obesity (BMl>35) & & -5.35 & $-7.57 ;-3.12$ & $<0.001$ \\
\hline Non-vertebral fracture & & -3.28 & $-6.32 ;-0.24$ & 0.034 \\
\hline Hypoestrogenism & & -2.61 & $-4.30 ;-0.92$ & 0.002 \\
antecedents & & -2.05 & $-3.97 ;-0.14$ & 0.036 \\
\hline Vertebral fracture & & 51.83 & $50.50 ; 53.15$ & $<0.001$ \\
\hline Constant & & & & \\
\hline
\end{tabular}

$\mathrm{n}=804 ; \mathrm{p}(\mathrm{F})<0.0001 ; \mathrm{r}^{2}=0.224 ;$ Adjusted $\mathrm{r}^{2}=0.215$. BMl: Body mass index. 95\%Cl: 95\% Confidence Interval. *Chronic conditions: corticoid treatment, gait abnormalities for any reason, cognitive or visual deficit, depression, gastrectomy, bowel resection, inflammatory bowel disease, thyroidectomy, diabetes mellitus, chronic liver diseases, chronic obstructive pulmonary disease, rheumatoid arthritis, chronic kidney failure and transplantation. 
Regarding the literature on the topic [5,7-10,12, $16,18,20-29,34]$, the accurate estimation of osteoporosis and VFX impact on HRQoL is difficult because the populations studied and the definitions and methods used are particularly heterogeneous: 1) Previous studies may have used population samples as in our study, but also samples from primary care patients -and, therefore with some health problems- or even samples from hospital outpatient rheumatology clinics with more severe patients; 2) fracture definitions vary from morphometric (using different techniques to identify and grade deformities) to patients' self-referred fractures or limited to VFX with clinical symptoms; 3 ) designs vary from crosssectional (prevalent fractures) to prospective (incident fractures) with different temporal distances between the fracture and the HRQoL instrument administration; 4) instruments used to measure HRQoL are very different and with different clinimetric properties, and 5) while VFX are more prevalent in aged people and a substantial proportion of these individuals may have clinically relevant co-morbidities and concomitant functional limitations, study analyses do not always take into account confounders, including comorbidities or osteoporotic fractures from other localizations (i.e., hip fractures). In general, this literature suggest that the more severe the vertebral fractures (clinical, incident, referred by patients, or with samples from specialized centres with more severe patients, multiple fractures) the higher the effect on HRQoL. On the contrary, in osteoporotic patients with no fractures or only mild prevalent morphometric fractures, the effect can be minimal. Our results are consistent with this interpretation, although mild morphometric fractures (Genant grade 1) seem to affect physical domains in very similar ways to moderate-severe fractures.

PCS and MCS scores (not always age-adjusted) from studies reporting these summary components from SF-36 or SF-12 surveys [18,23-29,34] are shown in Table 6. In general, the PCS score follows the behaviour described with few differences between women with or without VFX in the case of prevalent fractures in population studies and higher in selected samples with more severe patients or incident fractures. As in our study, MCS scores, with some exceptions, were not different between women with or without VFX.

Some of the factors associated with a lower physical HRQoL are similar to those described in other studies (age, chronic conditions, and antecedents of osteoporotic fracture). Obesity has also been related to a poorer physical (not mental) HRQoL [35]. We have not identified papers adjusting for hypoestrogenism antecedents in osteoporosis or VFX quality-of-life assessment. Although climacteric symptoms may have negative effects on both the physical and mental components of the HRQoL, women with hypoestrogenism antecedents would have more marked climacteric symptoms and could also have other health problems associated with HRQoL losses.

Table 6 Physical and Mental Component Summary scores in studies using the Medical Outcomes Study Survey Form.

\begin{tabular}{|c|c|c|c|c|c|c|c|c|}
\hline \multirow[t]{2}{*}{ Author } & \multirow[t]{2}{*}{ Country } & \multirow[t]{2}{*}{ VFX } & \multirow[t]{2}{*}{ Instrument } & \multicolumn{2}{|c|}{ PCS } & \multicolumn{2}{|c|}{ MCS } & \multirow[t]{2}{*}{ Comments } \\
\hline & & & & $\begin{array}{l}\text { Without } \\
\text { VFX }\end{array}$ & With VFX & $\begin{array}{l}\text { Without } \\
\text { VFX }\end{array}$ & With VFX & \\
\hline FRAVO study & Spain & Prevalent & SF12 & $45.3 ; 45.7$ & $41.6 ; 40.2$ & $45.6 ; 44.0$ & $46.3 ; 45.5$ & $\begin{array}{l}\text { Scores for mild and moderate-severe } \\
\text { VFX. }\end{array}$ \\
\hline Lai et al, 2010 [34] & China & Prevalent & SF36 & 14.3 & $14.1 ; 12.7$ & 27.8 & $27.7 ; 27.2$ & $\begin{array}{l}\text { Scores for morphometric and clinical } \\
\text { VFX.* }\end{array}$ \\
\hline $\begin{array}{l}\text { Van Schoor et al } \\
2005[18]\end{array}$ & Holland & Prevalent & SF12 & 50.0 & $\begin{array}{l}49.5 ; 50.8 \\
\quad 42.1\end{array}$ & 55.8 & $\begin{array}{l}55.6 ; 53.6 \\
55.0\end{array}$ & $\begin{array}{l}\text { Scores for mild, moderate and severe } \\
\text { VFX. }\end{array}$ \\
\hline $\begin{array}{l}\text { Cockerill et al, } 2004 \\
\text { [27] }\end{array}$ & Europe & $\begin{array}{l}\text { Prevalent } \\
\text { Incident }\end{array}$ & SF12 & 43.7 & $41.2(39.9)$ & 49.1 & $50.8(47.2)$ & Scores for incident VFX in brackets. \\
\hline $\begin{array}{l}\text { Hallberg et al, } 2004 \\
\text { [28] }\end{array}$ & Sweden & Incident & SF36 & 44.3 & $29.6(34.2)$ & 51.3 & $45.8(44.3)$ & $\begin{array}{l}\text { Scores } 2 \text { years after the incident VFX in } \\
\text { brackets. }\end{array}$ \\
\hline Falch et al, 2003 [29] & Norway & Incident & SF36 & 46.2 & 31.7 & 46.0 & 46.2 & Referred to hospital for clinical VFX \\
\hline $\begin{array}{l}\text { Adachi et al, } 2001 \\
\text { [23] }\end{array}$ & Canada & Prevalent & SF36 & 48 & 44 & 53 & 54 & Morphometric subclinical VFX. \\
\hline $\begin{array}{l}\text { Tosteson et al, } 2001 \\
{[24]}\end{array}$ & USA & Prevalent & SF36 & 47.1 & 40.1 & 53.6 & 54.7 & 45\% with clinical VFX. \\
\hline $\begin{array}{l}\text { Naves Diaz et al, } \\
2001 \text { [25] }\end{array}$ & Spain & Prevalent & SF36 & 50 & 47 & 50 & 48 & Population study \\
\hline Hall et al, 1999 [26] & Australia & Prevalent & SF36 & 48 & 36 & 54 & 50 & Referred to hospital for clinical VFX \\
\hline
\end{tabular}

PCS: Physical Component Summary Score; MCS: Mental Component Summary Score; SF12: Medical Outcomes Survey Short-Form 12; VFX: Vertebral Fracture. *PCS and MCS scores seem to use a non standardized range of values. 
Apart from contributing to the scarce data in Spain on HRQoL osteoporosis related, our study has other strengths. First, we use a population sample not dominated by more sick women as in studies using samples recruited in outpatient clinics or in clinical trials (typically, people at high risk of fracture). In fact, PCS and MCS scores of our weighted sample are practically identical to the SF-12 population values published for Spain [31]. Second, this is one of the larger population samples with both densitometric and spine $x$-ray evaluations. Third, assessment of VFX was carried out with standardized and reliable methods. Fourth, we used multivariate analysis with an extended set of covariables to control confounding.

The study also has several limitations. First, crosssectional design does not allow the establishment of causal relationships. VFX can be a causal factor of deterioration in physical HRQoL, but limitations in physical function can also causally contribute to VFX. Second, information on chronic conditions was selfreferred and although we use patient pharmacologic treatments to improve this data, figures are subject to the usual biases of data obtained from interviews. Third, our sample (broken up into four non-balanced groups and analyzed for several stratums of age, chronic conditions, etc.) has few observations in certain substratums of some groups (i.e. VFX in younger women) and some of the HRQoL estimations can be quite unstable. Therefore, HRQoL figures in the stratum-groups should be considered with caution, especially in the extreme stratums with fewer cases. Fourth, our questionnaire had no information about physical activity, a relevant variable that could have influence on osteoporosis, fractures and HRQoL. Fifth, our study used the SF12 questionnaire, a generic HRQoL measurement instrument that allow us to compare our results with many of the published studies on osteoporosis and other diseases, however it is also possible that this instrument was not responsive enough to detect small changes in HRQoL in osteoporotic patients.

After adjusting for confounding factors, our results indicate that HRQoL was significantly lower in women who have experienced prevalent osteoporotic vertebral fractures (as compared with women -osteoporotic or not- without fractures). The most clinically relevant impact on HRQoL occurred in the physical domains, with an attributable reduction of about $8 \%-10 \%$ in the PCS score. Although the clinical relevance of vertebral fracture has been well established for long time, these results are important for burden-of-disease and cost-ofillness studies, and also reinforce the need to reduce the underdiagnosis and undertreatment of these fractures.

\section{Additional material}

Additional file 1: Dropouts in the FRAVO Study. Dropouts by reason

and age groups.

\section{List of abbreviations}

(ECOS-16): Assessment of health-related quality of life in osteoporosis; (BMI): Body mass index; (DXA): Dual-energy X-ray; (EQ5D): EuroQol 5-D; (HRQoL): Health Related Quality of Life; (MOS-SF): Medical Outcomes Study Survey Form; (MCS): Mental component summary scale; (MANOVA): Multivariable Analysis of Variance; (OPAQ): Osteoporosis Assessment Questionnaire; (OQLQ): Osteoporosis Quality of Life Questionnaire; (OPTQoL): OsteoporosisTargeted Quality of Life Questionnaire; (PCS): Physical component summary scale; (QUALIOST): Quality of Life in Osteoporosis; (QUALEFFO): Quality of Life Questionnaire of the European Foundation for Osteoporosis; (VFX): Vertebral fractures.

\section{Acknowledgements}

Funded by the General Directorate for Health Organization, Evaluation and Research (Project 0018/2005) and the General Directorate for Public Health of the Ministry of Health of the Autonomous Government of Valencia, and a non-conditioned research grant from Sanofi-Aventis.

\section{Author details}

${ }^{1}$ Centro Superior de Investigación en Salud Pública (CSISP), Valencia, Spain. ${ }^{2}$ Centro de Salud de Nazaret, Agencia Valenciana de la Salud. Valencia, Spain. ${ }^{3}$ Centro de Salud de Villamarchante, Agencia Valenciana de la Salud. Valencia, Spain.

\section{Authors' contributions}

JSG, SP and GSG carry out the design of the study and contributed with intellectual input in the design of this paper. BRM and GSG developed the most part of the fieldwork. IH and GSG make the analysis and written the initial drafts. All authors contributed to the writing of the manuscript, corrected draft versions and approved the final version of the manuscript.

\section{Conflicts of interests}

None of the sponsors played any role in the study design, the collection, analysis or interpretation of data, the writing of the manuscript, or in the decision to submit it for publication.

\section{Received: 13 October 2010 Accepted: 6 April 2011}

Published: 6 April 2011

\section{References}

1. Johnell O, Kanis JA: An estimate of the worldwide prevalence and disability associated with osteoporotic fractures. Osteoporos Int 2006, 17(12):1726-33.

2. Majumdar SR, Kim N, Colman I, Chahal AM, Raymond G, Jen H, Siminoski KG, Hanley DA, Rowe $\mathrm{BH}$ : Incidental vertebral fractures discovered with chest radiography in the emergency department: prevalence, recognition, and osteoporosis management in a cohort of elderly patients. Arch Intern Med 2005, 165(8):905-9.

3. Delmas PD, van de Langerijt $L$, Watts NB, Eastell $R$, Genant $H$, Grauer A, Cahall DL, IMPACT Study Group: Underdiagnosis of vertebral fractures is a worldwide problem: the IMPACT study. J Bone Miner Res 2005, 20(4):557-63.

4. Guyatt GH, Adachi JD, Clifton J, Griffith LE, Epstein RS, Juniper EF: Quality of life issues in women with vertebral fractures due to osteoporosis. Arthritis Rheum 1993, 36(6):750-6.

5. Lips P, van Schoor NM: Quality of life in patients with osteoporosis. Osteoporos Int 2005, 16(5):447-55.

6. Lizán Tudela L, Badia Llach X: Quality of life evaluation for osteoporosis [In Spanish]. Aten Primaria 2003, 31(2):126-33.

7. Peasgood T, Herrmann K, Kanis JA, Brazier JE: An updated systematic review of Health State Utility Values for osteoporosis related conditions. Osteoporos Int 2009, 20(6):853-68. 
8. Cook DJ, Guyatt GH, Adachi JD, Epstein RS, Juniper EF, Austin PA, Clifton J Rosen CJ, Kessenich CR, Stock JL, Overdorf J, Miller PD, Erickson AL, McCLung MR, McClung BL, Griffith LE, loannidis G: Development and validation of the mini-osteoporosis quality of life questionnaire (OQLQ) in osteoporotic women with back pain due to vertebral fractures. Osteoporosis Quality of Life Study Group. Osteoporos Int 1999, 10(3):207-13.

9. Badia X, Díez-Pérez A, Alvarez-Sanz C, Díaz-López B, Diaz-Curiel M, Guillén F, González-Macias J, Spanish GRECO Study Group: Measuring quality of life in women with vertebral fractures due to osteoporosis: a comparison of the OQLQ and QUALEFFO. Qual Life Res 2001, 10(4):307-17.

10. Lips P, Cooper C, Agnusdei D, Caulin F, Egger P, Johnell O, Kanis JA, Kellingray S, Leplege A, Liberman UA, McCloskey E, Minne H, Reeve J, Reginster JY, Scholz M, Todd C, de Vernejoul MC, Wiklund I: Quality of life in patients with vertebral fractures: validation of the Quality of Life Questionnaire of the European Foundation for Osteoporosis (QUALEFFO). Working Party for Quality of Life of the European Foundation for Osteoporosis. Osteoporos Int 10(2):150-60.

11. Randell AG, Bhalerao N, Nguyen TV, Sambrook PN, Eisman JA, Silverman SL: Quality of life in osteoporosis: reliability, consistency, and validity of the Osteoporosis Assessment Questionnaire. J Rheumatol 1998, 25(6):1171-9.

12. Cantarelli FB, Szejnfeld VL, Oliveira LM, Ciconelli RM, Ferraz MB: Quality of life in patients with osteoporosis fractures: cultural adaptation, reliability and validity of the Osteoporosis Assessment Questionnaire. Clin Exp Rheumatol 1999, 17(5):547-51.

13. Lydick E, Zimmerman SI, Yawn B, Love B, Kleerekoper M, Ross P, Martin A, Holmes R: Development and validation of a discriminative quality of life questionnaire for osteoporosis (the OPTQoL). J Bone Miner Res 1997, 12(3):456-63.

14. Chandler JM, Martin AR, Girman C, Ross PD, Love-McClung B, Lydick E, Yawn BP: Reliability of an Osteoporosis-Targeted Quality of Life Survey Instrument for use in the community: OPTQoL. Osteoporos Int 1998, 8(2):127-35.

15. Badia X, Prieto L, Roset M, Díez-Pérez A: Development of the ECOS-16 clinical questionnaire for the assessment of the quality of life in patients with osteoporosis [In Spanish]. Med Clin (Barc) 2000, 114(Suppl 3):68-75.

16. Adachi JD, Adami S, Gehlbach S, Anderson FA, Boonen S, Chapurlat RD, Compston JE, Cooper C, Delmas P, Díez-Pérez A, Greenspan SL, Hooven FH, LaCroix AZ, Lindsay R, Netelenbos JC, Wu O, Pfeilschifter J, Roux C, Saag KG, Sambrook PN, Silverman S, Siris ES, Nika G, Watts NB, GLOW Investigators: Impact of Prevalent Fractures on Quality of Life: Baseline Results From the Global Longitudinal Study of Osteoporosis in Women. Mayo Clin Proc 2010, 85(9):806-13.

17. van Schoor NM, Yu H, Bobula J, Lips P: Cross-geographic region differences in quality of life in women with and without vertebral fracture. Osteoporos Int 2009, 20(10):1759-66.

18. van Schoor NM, Smit JH, Twisk JW, Lips P: Impact of vertebral deformities, osteoarthritis, and other chronic diseases on quality of life: a populationbased study. Osteoporos Int 2005, 16(7):749-56.

19. Marquis $P$, Cialdella $P$, De la Loge C: Development and validation of a specific quality of life module in post-menopausal women with osteoporosis: the QUALIOST. Qual Life Res 2001, 10(6):555-66.

20. de la Loge C, Sullivan K, Pinkney R, Marquis P, Roux C, Meunier PJ: Crosscultural validation and analysis of responsiveness of the QUALIOST: QUAlity of Life questionnaire In OSTeoporosis. Health Qual Life Outcomes 2005, 3:69.

21. Adachi JD, loannidis G, Pickard L, Berger C, Prior JC, Joseph L, Hanley DA, Olszynski WP, Murray TM, Anastassiades T, Hopman W, Brown JP, Kirkland S, Joyce C, Papaioannou A, Poliquin S, Tenenhouse A, Papadimitropoulos EA: The association between osteoporotic fractures and health-related quality of life as measured by the Health Utilities Index in the Canadian Multicentre Osteoporosis Study (CaMos). Osteoporos Int 2003, 14(11):895-904.

22. Gold DT: The clinical impact of vertebral fractures: quality of life in women with osteoporosis. Bone 1996, 18(3 Suppl):185S-189S.

23. Adachi JD, loannidis G, Berger C, Joseph L, Papaioannou A, Pickard L, Papadimitropoulos EA, Hopman W, Poliquin S, Prior JC, Hanley DA, Olszynski WP, Anastassiades T, Brown JP, Murray T, Jackson SA, Tenenhouse A, Canadian Multicentre Osteoporosis Study (CaMos) Research Group: The influence of osteoporotic fractures on health-related quality of life in community-dwelling men and women across Canada. Osteoporos Int 2001, 12(11):903-8.

24. Tosteson AN, Gabriel SE, Grove MR, Moncur MM, Kneeland TS, Melton LJ: Impact of hip and vertebral fractures on quality-adjusted life years. Osteoporos Int 2001, 12(12):1042-9.

25. Naves Díaz M, Díaz López JB, Rodríguez Rebollar A, Gómez Alonso C, Díaz Corte C, Cannata Andía J: Effect of vertebral fracture on health related quality of life in a Spanish population older than 54 years [in Spanish]. Med Clin (Barc) 2001, 116(14):533-5.

26. Hall SE, Criddle RA, Comito TL, Prince RL: A case-control study of quality of life and functional impairment in women with long-standing vertebral osteoporotic fracture. Osteoporos Int 1999, 9(6):508-15.

27. Cockerill W, Lunt M, Silman AJ, Cooper C, Lips P, Bhalla AK, Cannata JB, Eastell R, Felsenberg D, Gennari C, Johnell O, Kanis JA, Kiss C, Masaryk P, Naves M, Poor G, Raspe H, Reid DM, Reeve J, Stepan J, Todd C, Woolf AD, O'Neill TW: Health-related quality of life and radiographic vertebral fracture. Osteoporos Int 2004, 15(2):113-9.

28. Hallberg I, Bachrach-Lindström M, Hammerby S, Toss G, Ek AC: Healthrelated quality of life after vertebral or hip fracture: a seven-year followup study. BMC Musculoskelet Disord 2009, 10:135.

29. Falch JA, Bentzen $H$, Dahl AA: Pain, functional level and emotional problems of women with osteoporosis and vertebral fractures [in Norwegian]. Tidsskr Nor Laegeforen 2003, 123(23):3355-7.

30. Sanfélix-Genovés J, Reig-Molla B, Sanfélix-Gimeno G, Peiró S, GraellsFerrer M, Vega-Martínez M, Giner V: The population-based prevalence of osteoporotic vertebral fracture and densitometric osteoporosis in postmenopausal women over 50 in Valencia, Spain (the FRAVO Study). Bone 2010, 47(3):610-6.

31. Vilagut G, Valderas JM, Ferrer M, Garin O, López-García E, Alonso J: Interpretation of SF-36 and SF-12 questionnaires in Spain: physical and mental components [in Spanish]. Med Clin (Barc) 2008, 130(19):726-35.

32. Genant HK, Wu CY, van Kuijk C, Nevitt MC: Vertebral fracture assessment using a semiquantitative technique. J Bone Miner Res 1993, 8:1137-48.

33. Naves M, Díaz-López JB, Gómez C, Rodríguez-Rebollar A, RodríguezGarcía M, Cannata-Andía JB: The effect of vertebral fracture as a risk factor for osteoporotic fracture and mortality in a Spanish population. Osteoporos Int 2003, 14:520-4.

34. Lai BM, Tsang SW, Lam CL, Kung AW: Validation of the quality of life questionnaire of the European foundation for osteoporosis (QUALEFFO31) in Chinese. Clin Rheumatol 2010, 29(9):965-72.

35. Hopman WM, Berger C, Joseph L, Barr SI, Gao Y, Prior JC, Poliquin S, Towheed T, Anastassiades T, CaMos Research Group: The association between body mass index and health-related quality of life: data from CaMos, a stratified population study. Qual Life Res 2007, 16(10):1595-603.

\section{doi:10.1186/1477-7525-9-20}

Cite this article as: Sanfélix-Genovés et al:: Impact of osteoporosis and vertebral fractures on quality-of-life. a population-based study in Valencia, Spain (The FRAVO Study). Health and Quality of Life Outcomes 2011 9:20.

\section{Submit your next manuscript to BioMed Central and take full advantage of:}

- Convenient online submission

- Thorough peer review

- No space constraints or color figure charges

- Immediate publication on acceptance

- Inclusion in PubMed, CAS, Scopus and Google Scholar

- Research which is freely available for redistribution

Submit your manuscript at www.biomedcentral.com/submit
C Biomed Central 